\title{
Un monde diffracté. Théories joola du double
} animal

A diffracted world: Diola theories of the animal double

Odile Journet-Diallo

\section{OpenEdition}

1 Journals

Édition électronique

URL : http://journals.openedition.org/span/1598

DOI : 10.4000/span. 1598

ISSN : 2268-1558

Éditeur

École pratique des hautes études. Sciences humaines

\section{Édition imprimée}

Date de publication : 1 décembre 1998

Pagination : 203-230

ISSN : 0294-7080

\section{Référence électronique}

Odile Journet-Diallo, « Un monde diffracté. Théories joola du double animal », Systèmes de pensée en

Afrique noire [En ligne], 15 | 1998, mis en ligne le 04 juin 2014, consulté le 19 avril 2019. URL : http:// journals.openedition.org/span/1598; DOI : 10.4000/span.1598 


\section{UN MONDE DIFFRACTÉ \\ Théories joola du double animal}

\section{par}

\section{Odile Journet-Diallo}

"Il y avait une femme au village de Niaban qui avait pour double un énorme hippopotame. Celui-ci était venu dans nos rizières à Esanal. On avait décidé de le prendre. On l'a encerclé. Au milieu de la nuit, à Niaban, la fermme s'est réveillée en burlant: "allez vite à Esana, ils m'ont encerclée, ils vont me tuer!". Les hommes de Niaban sont partis pour venir ici, mais lorsqu'ils sont arrivés, ils ont entendu résonner le gros tambour à lères annonçant la mort de l'bippopotame. A leur retour au village, la femme était décédée. "

Esana, chroniques villageoises, 1993

La notion de double animal chez les Joola du sud du Sénégal et du nord de la Guinée-Bissau ne semble guère, a priori, pouvoir alimenter le débat sur la pertinence de l'usage du terme de totémisme appliqué aux sociétés africaines. Car il s'agit là d'une conception qui parait relever beaucoup plus évidemment des théories de la personne que d'une manière de rendre compte des différenciations sociales : avant que d'être métaphorique, la relation au double animal est d'abord posée comme une relation ontologique. A chaque humain est associé un alter animal,

${ }^{1}$ Niaban et Esana sont deux villages du pays felup (ou "ajaamat ") au nord-ouest de la Guinée-Bissau, distants d'une dizaine de kilomètres.

\section{Totémismes}

Systèmes de pensée en Afrique noire, 15, 1998 
composante intime de la personne. Constituer ce double en espèce totémique pouvait paraître aventureux. S'il n'avait existé un précédent, à savoir l'article de J. D. Sapir (1977) qui développait, à propos de la notion de double animal chez les Kujamaat, un groupe joola de la région du Fogny (nord de la Casamance), l'idée d'un " totémisme complémentaire ", j'aurais hésité à faire le lien entre ces deux champs. Les matériaux recueillis par J. D. Sapir constituaient au demeurant une variante qui semblait trop éloignée de ceux qui m'étaient familiers, dans les régions plus méridionales, pour pouvoir d'emblée le suivre dans sa démonstration. Comment, dans d'autres groupes joola, et notamment chez les Felup de Guinée-Bissau, comprendre la portée classificatoire du système des doubles animaux lorsqu'il s'agit de penser le monde social ? I fallait pour cela reprendre l'enquête sur des faits trop hâtivement classés.

\section{La notion commune d'ewuum}

Dans un article de 1973, P. Smith (1973) proposait de limiter la notion de "double" aux seuls cas définis par les trois conditions suivantes :

- que la personne et son double soient discontinus,

- qu'ils partagent une destinée commune ou une conscience commune, - que la personne et son double soient complémentaires l'un de l'autre. Les représentations joola du double animal (ewuum, pl. siwuumesu) répondent à ces conditions : le double animal est l'objectivation discontinue d'une part essentielle du moi. Sa destinée est entièrement liée à celle de son homologue humain comme en témoigne, parmi bien d'autres, le court récit rapporté ci-dessus.

Littéralement, ewuum signifierait : " résultat de transformation ". Dans plusieurs variantes dialectales, la racine $w u$ est communément utilisée comme verbe pour signifier " réincarner " (par exemple, awua, " celui qui se réincarne ", désignant le principe spirituel d'un défunt qui revient). Ewuum n'a cependant rien à voir avec l'animal en quoi peut se transformer un sorcier (alaghor, " celui qui se transforme "), même si, diton, les sorciers jouent de cette possible confusion. 
Les doubles empruntent toujours l'apparence d'animaux sauvages, à l'exception, dans certains groupes joola, de certains animaux d'élevage "bizarres ", telle une vache sans corne. Animaux du marigot, de la forêt ou de la brousse, ils sont réputés difficiles à capturer, soit qu'ils demeurent invisibles, soit qu'ils disparaissent dans un nuage de poussière à l'approche du chasseur, soit que le fusil ou l'arc pointé sur eux ne réagisse pas à la détente, soit encore que la balle ou la flèche ne réussisse pas à les transpercer. Mais si le double est blessé, son homologue humain subira une même atteinte au même endroit du corps. D'une personne évanouie, on dira qu'elle "a été attrapée à son ewuum ", que celui-ci a été immobilisé sur le point d'être tué, ou encore, s'il s'agit d'un double aquatique, qu'il a été laissé par la marée, accroché quelque part. Une parente, généralement une tante paternelle, parcourt le village en criant : "Lâchez mon neveu!". Si le double meurt, son alter meurt aussi, à moins d'une intervention rituelle spécifique et rapide.

A ce niveau de généralité, la notion d'ewuum est commune à l'ensemble des groupes joola de la Basse-Casamance et du nord de la Guinée-Bissau. Sa mise en rapport avec une unité sociale n'apparaît pas comme caractéristique immédiate. L'erwum peut d'autant moins être mis en rapport avec des divisions claniques qu'il apparait singulièrement difficile de raisonner en termes de clans. On peut trouver ça et là quelques traits permettant d'esquisser les contours d'unités sociales supralignagères qui se réfèrent à une origine commune, mais d'ordre géographique. Ces unités ne se manifestent pas par des formes de mobilisation rituelle, et leur existence ne constitue pas de système à l'intérieur duquel elles se différencieraient par des appellations, ou par référence à un récit de fondation.

Chez les Joola-Felup dont j'examinerai plus en détail le système des doubles, en l'absence de noms, d'interdits spécifiques ou de spécialisation qui leur correspondrait, aucune tradition historique ne permet de reconstituer des unités claniques. Les Felup ignorent les patronymes : les jeunes qui sont aujourd'hui dotés d'un nom de famille l'ont adopté de façon souvent arbitraire en puisant dans le stock des patronymes des villages joola sénégalais ou encore dans les noms portugais. Pour les plus âgés, l'opération de recensement national, au printemps 1994, m'avait donné 
l'occasion d'assister à quelques scènes rocambolesques d'adoption, sur le vif, d'un patronyme dans la file d'attente.

Dans la plupart des autres groupes joola, seuls les patronymes Jeeju, Sambu et Jaata sont liés à une spécialisation fonctionnelle : Jeeju désigne les lignages de forgerons (sans qu'ils soient fédérés au sein d'un même clan), Sambu (de sambunasu, "le feu ") et Jaata désignent des lignages liés au Jeeju dans les rituels relatifs à la forge. Mais chez les Felup, "Jeeju ", "Sambu ", "Jaata " sont souvent utilisés comme prénoms et, comme tels, ne renvoient nullement à cette implication. Quoi qu'il en soit, au vu des aléas qui président ici à l'affectation d'un patronyme et aux transformations dont il peut être l'objet, celui-ci, lorsqu'il existe, ne peut guère servir d'indicateur d'une quelconque appartenance clanique, voire même lignagère.

Le double-animal jouerait-il ce rôle ? Les données dont je disposais ne me permettaient guère de le penser. Dans l'exemple développé par J. D. Sapir, les espèces choisies comme siwuum ne sont nullement associées à tel ou tel clan ou tel ou tel lignage. Dans l'autre cas que j'évoquerai, celui des Joola " esulelu " (Pointe St-Georges, sur la rive sud de la Casamance), les membres de lignages de même nom peuvent avoir des doubles différents, alors que des individus de deux lignages ou segments de lignage non apparentés peuvent avoir un même animal pour ewuum.

Les faits joola ne relèvent pas assurément d'un " totémisme clanique ". Si l'on admet depuis Radcliffe-Brown que celui-ci ne soit qu'une variété du totémisme, le problème demeure toutefois entier. Car pour comprendre comment la notion d'ewuum peut révéler quelque chose de la façon dont les Joola se représentent leurs divisions sociales, il faudra postuler que le totémisme puisse également fonctionner sur d'autres modes que celui de l'homologie interne entre deux séries : unités sociales / objets ou espèces totémiques.

Avant d'entrer plus en détail dans l'exemple felup, j'évoquerai deux autres variantes : celle des Joola " kujamaat " (rive nord de la Casamance) à propos de laquelle J. D. Sapir développe l'analyse de ce qu'il appelle d'emblée un " double totémique "; et celle du groupe " esulelu ", sur lequel je disposais de quelques données recueillies de manière annexe, il 
y a plusieurs années. Il ne pourra s'agir d'une comparaison terme à terme, car, dans ces trois exemples, les matériaux utilisés ne sont équivalents ni dans leur contenu ni dans leur niveau d'élaboration. Mais ils permettront de souligner la portée heuristique du réexamen de la notion de totémisme que, faute d'en avoir exploré toutes les implications, je ne considérais pas spécialement pertinente lorsqu'il s'agissait d' « affaires " de doubles.

\section{L'exemple ajamaat (pl. kujamaat) $)^{2}$ un double excrété}

J. D. Sapir rapporte que chez les Kujamaat, le double animal est, un beau jour, excrété par son homologue humain sous forme, par exemple, d'un léopard, d'une antilope, d'un singe (en général pour les hommes), d'une antilope, d'un lézard ou d'un serpent (généralement pour les fermmes). L'important, souligne l'auteur, est de considérer le lieu où cet animal va chercher refuge : s'il s'agit du double d'un garçon, d'un homme ou d'une fille non encore mariée, il part dans le périmètre de la résidence du lignage agnatique de la mère de celui(celle)-ci. Mais lorsque la fille se marie, son double part vivre auprès de ou dans la maison de ses propres agnats. L'animal et son " producteur" sont unis de telle sorte que tout ce qui arrive à l'un arrive à l'autre. Personne et double, dit-il, désignent en même temps les deux moitiés d'une métonymie basée sur une communauté de sang et d'« âme \%. J. D. Sapir développe son analyse dans deux directions :

1 - il montre tout d'abord comment "l'idiome ewuum " permet de penser la dualité du moi et la dualité de ses appartenances. "To have a double in a very real sense, is to deny the physical fact that an individual can be only one self and at only one place, and to assert instead the social and psychological fact that an individual is always two selves at two places."

2 " ajamaat": "celui qui comprend" (le langage humain), i.e. la personne humaine. Le terme est aussi bien employé au sens de " natif ". D'autres groupes joola se désignent de la même façon, en particulier ceux que les Portugais ont appelés "Pelufes " devenu "Felup " (notre troisième exemple), et qui s'appellent eux-mêmes "Kujamutay". 
Cependant la relation individu-double est asymétrique et inégale : un ewuum est une forme d'excrément, quelque chose dont on s'est débarrassé, mais que l'on n'a pas éliminé. Plus exactement, c'est quelque chose qui est déplacé, mis à l'arrière-plan, de même que, pour avoir une personnalité sociale viable, l'individu qui a deux foyers doit mettre de côté, déplacer l'un en faveur de l'autre.

2 - Puis, de l'« idiome siwuum ", il développe deux modèles analogiques. A partir des permutations possibles de la relation entre une moitié (personne ou double) d'un moi et une moitié (personne ou double) d'un autre moi, le premier modèle fait apparaître quatre types de relations interpersonnelles. En effet, quand un Joola Ajamaat - et c'est aussi le cas dans nos autres exemples - dit : " A a parlé, ou fait telle chose à B ", cela ne veut pas seulement dire que l'humain $A$ a fait ceci à l'humain $B$, ou au double $B$, mais aussi que le double de $A$ a fait ceci à l'humain $B$, ou au double $B$. Les combinaisons possibles de cette relation humain-double circonscrivent les quatre rôles qu'un homme peut alternativement jouer vis-à-vis de ses parents :

- comme agnat (relation de personne physique à personne physique, relation de co-résidence agnatique, marquée par la compétition)

. comme neveu utérin (relation de double à personne physique, relation de familiarité)

- comme oncle maternel (relation de personne physique à double, relation de protection et d'aide)

. comme cousin parallèle matrilatéral (relation de double à double, chez l'oncle maternel, relation non compétitive).

S'il s'agit d'une femme, la même combinatoire permet de la situer tour à tour comme épouse, comme agnate mariée ailleurs, comme épouse de frère, comme sœur.

Sont par contre absents de ce jeu totémique les parents utérins d'une fernme mariée et les beaux-parents d'un homme (parents de son épouse et parents des maris de ses sœurs). Entre ces catégories les relations ne sont développées ni entre personnes physiques, ni entre doubles.

Ainsi les siwuum fournissent-ils aux Kujamaat le moyen de conceptualiser les aspects importants de leur vie sociale, en définissant comment des catégories de gens sont en rapport avec chaque autre. La projection de la métonymie personne/ewuum dans le champ des relations sociales 


\section{Un monde diffracté}

permet de rendre compte de la majorité des situations interpersonnelles. Sapir souligne en particulier l'analogie existant entre la position des sizerum qui cherchent refuge dans ou autour de l'enclos et celle de leurs homologues humains, kusunful (neveux utérins) et furimen (filles du lignage mariées ailleurs) : ces deux catégories cherchent un refuge " par rapport au huis clos où se déroule leur vie quotidienne et qui est si souvent caractérisé par la concurrence, la compétition au sujet des terres, la jalousie et la sorcellerie ".

11 décline enfin un deuxième modèle analogique en faisant jouer les variables : personnes physiques/doubles vs agnats (masculins et féminins), cousins parallèles matrilatéraux, épouses. Ce modèle met en relief, dit-il, l'ultime dualité du système entier : comme personne physique, un homme vit avec son père, ses frères et ses enfants; mais, comme double, il vit plutôt avec sa mère, les sœurs de sa mère et leurs enfants. Pour Sapir, ces représentations contribuent à mettre en évidence le poids de la matrifiliation dans une organisation sociale patrilinéaire. "Parler de siwuum, c'est dire que l'affaire est exclusivement un problème de kusunful (neveux utérins) ou de furimen (natives du lignage mariées ailleurs) ".

$\mathrm{Au}$ cours de son développement, J. D. Sapir décrit également un rituel appelé kajupen destiné à permettre à un malade - dont le double a été détruit ou blessé - de se retrouver en position de produire un nouveau double : le malade étreint une chèvre, ventre contre ventre. Le maitre du rituel frappe légèrement le dos de l'animal, qui sera ensuite immolé, afin de transférer le yut (l'« âme sang ") de l'animal au malade.

Appliquée aux faits kujamaat, le grand intérêt de cette démonstration est sans aucun doute de faire comprendre comment l'ewnum permet de penser les différenciations et les oppositions non pas entre unités discontinues d'unifiliation, mais au sein même de la parenté, en faisant notarnment ressortir le caractère toujours problématique de la corésidence. A un niveau plus général, elle invite à centrer l'attention non pas tant sur les rapports entre espèces animales en tant que telles, mais sur les lieux où elles résident. 
L'exemple esulelu (Joola de la Pointe St-Georges) ${ }^{3}:$ un double dédoublé

L'idée de cette dualité du moi mise en évidence par J. D. Sapir s'exprime, dans le groupe esulelu, par une figure bien particulière puisqu'ici, c'est le double lui-même qui se dédouble. A chaque patrilignage est associé un ewuum spécifique que seules les furimen, les agnates mariées ailleurs, ont la faculté de reconnaître. Dans la liste des espèces en lesquelles pouvaient s'incarner un ewuum, Sapir mentionnait, dans l'exemple kujamaat, l'antilope, le léopard, le singe, le serpent, le lézard, et de façon beaucoup plus rare, l'hyène ou le crocodile. Le seul oiseau qui lui avait jamais été mentionné était le vautour, et le seul poisson, un petit poisson des rizières. Chez les Esulelu, qui vivent au bord de l'eau, on trouve, outre les espèces signalées, de nombreuses espèces aquatiques: apparaissent dans la série le mulet, le requin, le crocodile, beaucoup plus fréquemment mentionné, et l'hippopotame (ekaw, " la masse "). Figurent aussi dans la liste des serpents venimeux, comme la vipère, que l'on évitera de tuer. Sont exclus de la liste les insectes. La plupart du temps, ces animaux demeurent invisibles et se révèlent très difficiles à capturer. Le peu de relations que les humains puissent entretenir avec ces siwuum sont des relations de protection et de respect réciproque : les furimen mettent en garde les chasseurs ou les pêcheurs trop acharnés. Des interdits de lieux protègent aussi les siwuum : telle cette zone, à la confluence du marigot de Kamobeul et de la Casamance, où il est interdit de pêcher. A cet endroit, au demeurant dangereux pour la navigation, on raconte d'ailleurs que des hippopotames ont fréquemment sauvé de la noyade des piroguiers qui avaient chaviré. Cependant, il n'y a pas d'interdit alimentaire sur l'espèce animale liée à tel ou tel lignage : ceux qui ont pour ewuum le poisson mulet en pêchent et en mangent. Car sous l'apparence du mulet, l'ewuum n'est pas un poisson ordinaire et sait se dissimuler.

Mais l'ewnum esulelu se dédouble sous la forme d'un varan de petite taille, de type " gueule tapée ", appelé jibalas (" petite gueule tapée "). "Jibalas" est devenu, chez les Esulelu, le terme générique désignant le

3 " esulelu", "le village de fond ", nom donné par les Joola qui habitent plus au sud. 
double animal. Hypostase de ewuum, jibalas prend le devant de la scène. "C'est à lui que l'on pense lorsque l'on parle d'ewuum " précisent les informateurs. Quel que soit le statut ou le sexe de son homologue humain, jibalas réside chez les oncles maternels. Il est fortement impliqué dans la vie quotidienne et rituelle. Tout adulte veille à ce que personne ne tue ces petits sauriens que l'on distingue aisément des " gueules tapées " ordinaires qui avalent les poussins. Ils sont couramment évoqués pour traiter des bagarres entre enfants : " oui, on vous a bien vus, si vous continuez, vous détruirez quelque chose". Menacer de maltraiter le jibalas d'un neveu est également, pour l'oncle maternel, le seul mode de contrainte qu'il puisse exercer à son encontre : « Tu refuses de m'écouter, tu verras, je vais te (i.e. ton jibalas) sortir, te laisser sécher au soleil et bien te battre! ".

Non moins remarquable est l'intervention de jibalas dans les rituels de naissance et de funérailles : à la naissance de chacun de leurs enfants, les furimen reviennent chez leur père pour faire un sacrifice de coq et de vin de palme sur le bákin ${ }^{4}$ ufila (autel lignager) de leur maison natale. Ce sacrifice porte un nom spécial, këhiit, que les Chrétiens et les Musulmans ont tôt fait de traduire par " baptême ". Je n'ai malheureusement pas eu l'occasion d'assister à ce rituel chez les Esulelu : il s'agit de confier le double de l'enfant au ufila, et, par extension à ses oncles maternels. D'autres sacrifices auront lieu au ufila, à chaque étape du développement de l'enfant (marche, sevrage, etc.). Si ces rituels sont négligés, le jibalas de l'enfant, dit-on, " va errer seul, comme perdu ". L'enfant tombe malade. On dit alors: " jibalas ol ajo", " son double est sorti ". Un sacrifice au ufila doit permettre de le récupérer, puisque c'est le bákin qui l'a fait sortir qui doit le reprendre.

Au moment des funérailles, le troisième ou quatrième jour après l'enterrement, a lieu un sacrifice qu'on appelle kanonen et qui signe la séparation définitive du mort d'avec les vivants. Ce sacrifice a lieu après une visite au cimetière lors de laquelle les neveux utérins, munis d'un

${ }^{4}$ Le terme bákin (pl. ukin) désigne à la fois une puissance intermédiaire entre Dieu (Emitey) et les humains, l'autel, le sanctuaire. Tous les groupes joola ne parlent pas de bákin à propos des autels lignagers, certains leur réservant le terme de utilaw (cf. infra). 
coq, ont allumé un feu et effectué des libations de vin de palme et d'eau enfarinée sur la tombe. De retour, ils égorgent le coq dans la chambre du mort, puis le déposent sur l'autel lignager. Le kanonen " commence le mort ", en permettant au défunt d'accéder au usanjol ("l'abreuvoir"). Le sacrifice et les libations du kanonen sont effectués en parallèle chez l'oncle maternel : car, pour " commencer le mort ", il faut que son double soit lui aussi explicitement congédié. A la fin du sacrifice, c'est en ces termes que s'exprime le responsable du ufila : « ewuumey ol eje ", " son double est parti ", ou encore : « ajo kabakol ", "il part /son enterrement ", i.e., il part avec tout son bien, double compris.

Le rituel kajupen qu'évoquait Sapir existe aussi chez les Esulelu, pour redonner vie à quelqu'un qui est au bord de la mort : ce rituel s'effectue très discrètement avec un bœuf que l'on égorge et dont on transfère le yal (équivalent de cette " âme sang » des Kujamaat) à la victime. Ce transfert est supposé redonner également vie au jibalas.

Si l'on développait le même modèle analogique que J. D. Sapir au niveau des relations interpersonnelles, nous retrouverions, définis par le rapport humain/jibalas, les quatre rôles qu'un homme peut alternativement jouer vis-à-vis de ses parents, à cette différence près que les relations entre cousins parallèles matrilatéraux - kusunful (neveux utérins) dont les doubles cohabitent chez l'oncle - sont considérées ici comme fortes et compétitives et non comme sporadiques et relativement indifférentes (les jibalas sont d'une même espèce, ce que ne sont pas les siwuum vivant ensemble chez les Kujamaat). Du côté des femmes, le système esulelu diffère notoirement du précédent puisque, lorsqu'elles se marient, leur jibalas reste dans le patrilignage de leur mère. Les doubles de la mère et du fils ne cohabitent pas. Mais nous ne pouvons pour l'instant guère aller plus loin dans l'interprétation du système esulelu en tant que forme de représentation des relations intra-familiales.

On notera cependant que, sous la figure d'une double incarnation du double, ce modèle opère la distinction entre un système formel de différenciation des patrilignages par l'ewuum (qui n'est pas identifié par son mode de résidence) et une relation substantielle et vitale entre un humain et son jibalas (spécifié par sa résidence). A l'instar de l'exemple ajamaat, c'est la relation agnats/utérins qui est mise en exergue dans 


\section{Un monde diffracté}

l'énoncé général du système, mais sous une autre variante : quel que soit son sexe ou son statut matrimonial, la vie d'une personne est toujours entre les mains du patrilignage de sa mère.

L'exemple ajaamat
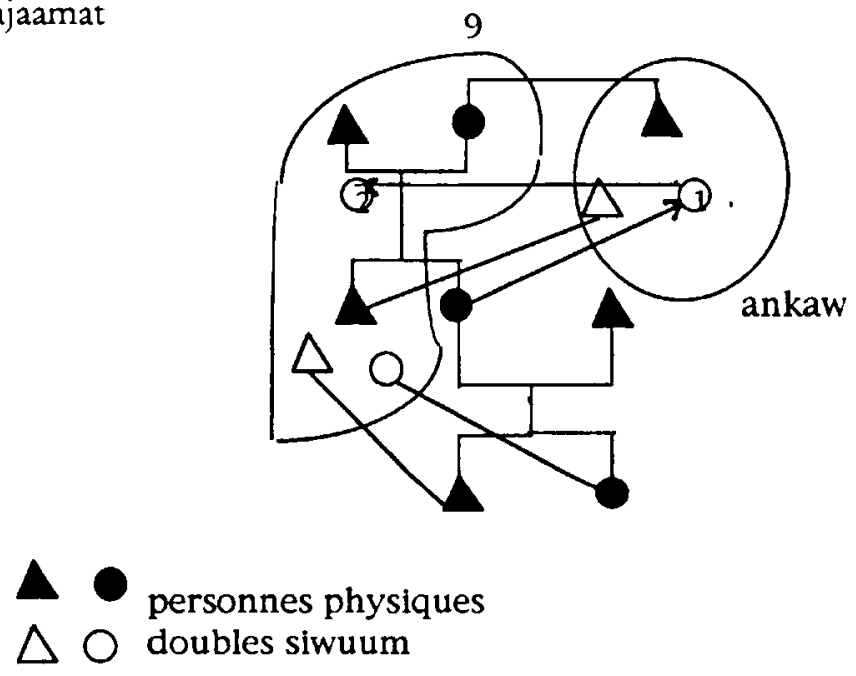

L'exemple esulelu

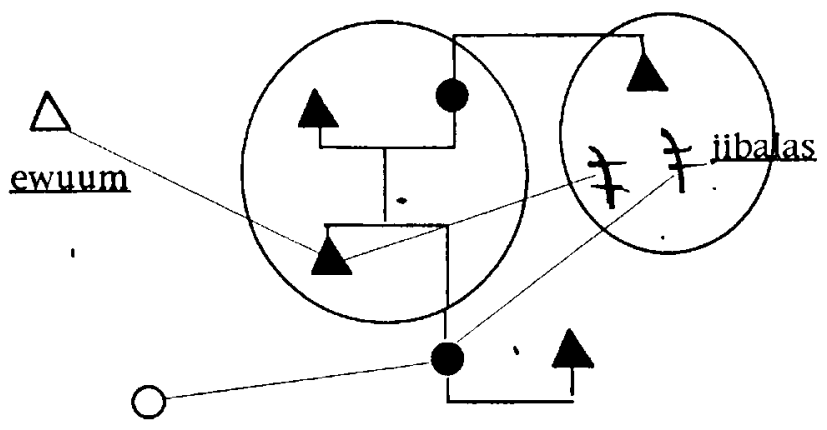




\section{L'exemple felup : des doubles démultipliés}

Avant de reprendre une enquête plus serrée sur les représentations du double animal en pays felup, $j$ 'avais plus d'une fois recueilli les récits d'épisodes mettant en scène la relation intime qui pouvait exister entre une personne mal en point et un animal chassé ou blessé.

« Il y avait un gars du village qui était excédé par un hippopotame qui venait ravager ses rizières chaque fois qu'il avait le dos tourné. Il est alors parti en pleine nuit et a essayé de le tirer au fusil... Le coup n'est pas parti. Il s'est alors précipité sur l'animal avec son coupe-coupe et l'a frappé jusqu'à ce qu'ils arrivent au marigot où l'hippopotame a disparu. Le lendemain, un autre homme est arrivé au dispensaire couvert de blessures. Personne n'a pu soigner correctement ses plaies. L'homme est allé consulter un clairvoyant qui lui a dit de partir chez le premier cultivateur avec une noix de bukunumabu ${ }^{5}$ afin de lui demander de lui faire kepuleenaku' ${ }^{6}$ : c'est vrai, j'ai déconné dans tes rizières et tu m'as blessé ". Ils sont allés sacrifier à l'autel de double de la maison, mais l'homme est quand même mort, car il fallait aussi faire le jugement public ".

L'enquête se révéla plus épineuse que je ne l'avais prévue. Si de tels récits abondent, les Felup, comme les autres Joola, répugnent à parler de leurs propres doubles. Quant aux principes généraux, ils me furent livrés dans un premier temps de manière extrêmement contradictoire.

\section{Identification des doubles}

Les espèces animales mentionnées comme siwuum potentiels vivent dans la mer, les marigots ou leurs abords (requin, baleine, poissons de diverses espèces, hippopotame, crocodile, loutre) ; dans la forêt ou la brousse (éléphant, buffle, antilope, panthère, hyène, singe, python,

\section{${ }^{5}$ carapa procera}

${ }^{6}$ Le kepuleen est un geste rituel qui consiste à souffler, en pulvérisant quelques gouttes de salive ou de vin de palme, sur divers segments du corps de l'interlocuteur. Omniprésent dans la pratique cultuelle, comme geste inscrivant le sacrifiant dans l'espace de protection d'un bákin, il acquiert un pouvoir spécifique en cas de conflit entre individus. L'offensé, sollicité par l'offenseur, mâche la noix de bukunumabu, dont il lui recrache la pulpe au visage. Ce geste doit " effacer de la bouche dont elle est sortie " la parole d'imprécation de l'offensé et suspendre l'affaire jusqu'à ce qu'elle soit jugée publiquement. 
serpent cracheur) ; dans les arbres (aigle, grue). A la différence des exemples précédents, le charognard n'en fait pas partie, ni aucune espèce de lézard.

De cette liste se dégage une caractéristique commune à la plupart de ces espèces : les animaux en question ne mettent ordinairement au monde qu'un seul rejeton à la fois. En ce qui concerne les exceptions à cette règle, comme c'est par exemple le cas du crocodile, les Felup précisent : * il y en a deux sortes: les ordinaires qui vont pondre leurs œufs dans les rizières près du marigot et les siwuum qui vont accoucher au fond de l'eau d'un seul enfant ". L'ensemble des siwuum circonscrit par ailleurs la totalité des éléments de l'environnement : l'eau, la terre (brousse et forêt), les airs. Ils jouent de ce point de vue un rôle régulateur : on sait par exemple qu'il est dangereux de récolter en une fois une trop grande quantité d'huîtres. Les doubles qui s'en nourrissent risquent d'attraper et de noyer le pêcheur impénitent. Cette dimension du totémisme, M. Dupire (1991) la soulignait à propos des Sereer Ndut qui, disait-elle, incluent dans un même système de représentations apparentement aux différents éléments de l'environnement et maitrise de ceux-ci : " du contrôle rituel de la nature, on passe insensiblement à sa protection ".

Ewuum nait dans la brousse ou dans le marigot quelques jours, voire quelques semaines, avant son homologue humain. Leur destinée est si intimement liée que dans toute discussion où il est question de doubles, les locuteurs ne précisent plus s'il s'agit de l'ewuum ou de son alter humain (anaw) : "Nous l'avons cherché dans le puits "... "J'étais tombée dans la mare *, etc.

De manière générale, il y a toujours précession des doubles sur les humains. Tout ce qui advient dans la vie individuelle ou sociale d'une personne est déjà advenu dans le monde des doubles. Ainsi, qu'il s'agisse de mariage ou de relations sexuelles, il faut, disent nos informateurs, " que leurs doubles se soient déjà aimés ". Cet étroit parallélisme suppose que l'on ne puisse s'unir qu'entre humains possédant un double de même espèce. "Comment une panthère pourrait-elle aimer une antilope!". Cette évidence felup m'avait d'abord plongée dans la perplexité compte tenu de l'extension des prohibitions matrimoniales et du nombre d'espèces animales fournissant des doubles aux habitants d'un seul village. 
Sachant que chaque patrilignage possède un autel (utilaw) associé à une espèce animale particulière, il y avait là une énigme. Laquelle fut au demeurant vite levée lorsque je compris enfin que chaque individu ne possédait pas un seul double, dédoublé ou non, mais une pluralité de doubles, de quatre à six, parfois plus. "Comme tu as plusieurs doigts, tu as plusieurs doubles". Un homme fort (ata sembe) est celui qui dispose de doubles nombreux, évoluant dans les différents espaces de la brousse, du marigot, des airs.

Cette démultiplication des doubles, si elle répondait à de premières questions, en posait évidemment de nouvelles.

L'identité de l'un des doubles d'un individu, directement transmise par ses géniteurs, est en principe bien connue puisque c'est celle à laquelle chaque lignage est attaché par ce petit autel où s'effectuent les rituels destinés à soigner les maladies provoquées par cette espèce. Cet autel, utilaw, n'est pas forcément implanté dans l'unité de résidence (ankaw) où l'on habite, compte tenu de la dispersion fréquente des segments de lignage entre plusieurs siank $k^{7}$.

Des différentes études menées sur les Joola, se dégage un schéma idéal qui a sans doute quelque pertinence dans les villages de BasseCasamance, mais ne peut être retenu pour les Felup : pour la plupart des observateurs, le ankaw correspondrait à un segment de lignage regroupant les ménages d'un homme et de trois à quatre de ses fils ; plusieurs siank constitueraient un sous-quartier; le quartier serait enfin la réunion de trois ou quatre lignages. Dans ce schéma idéal, même nuancé par la prise en compte d' "adoptions" en cas d'extinction du ankaw, unités de filiation et unités de résidence se recouperaient. Rien de tel dans l'exemple felup. Au terme d'une enquête, au demeurant laborieuse, il s'est avéré qu'à deux exceptions près, aucun des trente-sept siank du village de Esana n'était formé d'un unique segment de lignage. Chacun comprend des membres de lignages divers, mêlant autochtones

${ }^{7}$ Unité de résidence regroupant cinq à six maisons, le ankaw (pl. siank) est doté d'une forte individualité : chacun a son nom, ses surnoms, et il apparaît sur la scène rituelle comme une unité. 
et étrangers d'origine. Les segments de lignage sont ainsi dispersés entre différentes unités de résidence.

Dans l'ensemble de ces siank, seize autels de doubles (utilaw) sont implantés. Deux espèces animales sont représentées deux fois (eñeriñey, l'hyène et eluney, le serpent cracheur), mais correspondent dans les deux cas à des lignages non apparentés. Cependant, tous les lignages représentés dans le village n'y ont pas forcément leur utilaw. Certains l'ont laissé à son emplacement d'origine ou bien ont accepté d'en laisser la garde à un segment du même lignage installé dans un autre village.

Le commun des villageois ne connaît ordinairement que l'ewuum dont l'autel est entretenu par son patrilignage. Mais cet ewuum n'est pas forcément celui avec lequel il aura le plus d'affinité, celui avec lequel ses attaches seront les plus fortes (" ewuumey janu jumi jak jak ", "le double que tu vois bien, bien").

Soit un exemple : un homme et une femme mariés peuvent avoir chacun un patrimoine de doubles - qu'ils ne connaissent pas forcément dans lequel ils auront en commun deux ou trois siwuumesu de même espèce. L'homme issu d'un lignage associé à eñogay (hippopotame) sait qu'il a au moins un double eñogay. Sa femme, originaire d'un lignage associé à eñeriñey (hyène) sait qu'elle a au moins un double hyène. Puisqu'ils ont pu se marier, il se peut que la femme ait aussi un double hippopotame, ou que le mari ait aussi un double hyène. Mais il est tout aussi possible que les premiers de leurs doubles à s'unir aient été par exemple leurs doubles pythons.

Dans cette deuxième hypothèse, leur premier enfant aura pour double principal un python, mais les enfants suivants pourront en avoir un autre.

Ainsi un individu peut-il être lié beaucoup plus fortement à un ewuum autre que celui dont son patrilignage possède le utilaw: sans qu'ils le sachent, cet ewuum privilégié faisait partie du patrimoine de ses géniteurs. Ces doubles dont on ignore l'existence peuvent se révéler, au cours de la vie, par une affection, une blessure, une maladie. Ainsi de cet homme qui se débattait, soudainement pris de suffocation. Des pêcheurs étaient à ce moment-là en train de retirer leur filet ; ils avaient pris un certain poisson que, pour une raison ou une autre, ils ont rejeté à l'eau. 
L'homme fut soudainement apaisé et, au retour des pêcheurs, il alla " se confier " à l'autel du double poisson. Ou encore celui-ci qui, pris à la gorge, toussait, essayait en vain de cracher. Sur l'indication d'un clairvoyant (ana juke, "celui qui voit"), il part au utilaw de ioney, le crocodile, qui, lui dit-on, avait avalé trop goulûment des poissons et gardait des arêtes coincées dans la gorge. Certains siwuum se manifestent fréquemment par des troubles du comportement chez l'enfant : ainsi de celui qui pleurniche sans arrêt, qui frappe sa mère lorsqu'elle le prend, qui ne mange pas, on dira que son double est ailleurs, dans un ankaw où il n'est pas pris en charge. Ses parents iront là-bas pour y faire les rituels appropriés. Dans le cas d'un enfant pleurnicheur, on s'orientera vers un utilaw de hyène ; si l'enfant ne mange pas, vers celui du python.

On raconte aussi que les doubles, entre eux, peuvent se jouer des tours afin de nuire à certains humains : certains se transforment en individus de sexe opposé. Un double femelle prend ainsi l'apparence d'un mâle pour aller copuler avec un autre double femelle. L'homologue humain de cette dernière ne pourra jamais avoir d'enfants.

\section{Interdits et rituels liés aux doubles}

Hormis l'interdit - très général dans nombre de sociétés africaines qui pèse sur le marsouin, il n'y a pas d'espèce animale qui puisse être considérée chez les Joola comme véritablement ñiñi (interdite, dangereuse), qu'il s'agisse de la tuer ou de la manger. L'hippopotame, remarquable parmi les siwuum, on le mange sauf ceux qui savent en avoir un pour double. Les seuls véritables interdits alimentaires sont des interdits liés au sacrifice, qu'ils concernent viande, boisson ou toute autre nourriture consacrée à tel ou tel bákin. En matière de doubles, c'est plutôt la crainte de se faire du mal, de faire du mal voire de tuer un de ses enfants qui prédomine. Lorsque des parents apprennent que leur enfant possède tel ou tel double, ils cessent de chasser l'espèce en question et conseillent à leurs fils d'éviter de la tuer. Mais cette règle d'évitement n'est pas particulière à un lignage, ni partagée par tous les membres de ce lignage.

En revanche, la mise à mort d'un hippopotame - presque toujours considéré comme ewuum - est traitée rituellement comme un meurtre. Avant de prendre la décision de le chasser, les hommes du quartier se 


\section{Un monde diffracté}

réunissent et sacrifient aux principaux ukin réservés aux hommes : këyëku, bákin de la royauté, kareñaku, bákin de l'initiation, kañagenaku, "l'arc ", bákin de la chasse et de la guerre, bulampanabu, bákin de protection du village, mais aussi au karaayaku, grand bákin des femmes, lié à la fertilité. Le jour dit, une tranchée servant de piège est creusée. Lorsqu'il s'y retrouve prisonnier, l'hippopotame est tué au harpon, à l'arc et au fusil. Il est retiré du fossé et découpé sur place. Mais dès leur retour sur la place du quartier, les hommes font battre le gros tambour à lèvres et organisent les ñikulay, danses que l'on pratique lors des funérailles de toute personne adulte. Le premier chasseur à avoir atteint l'animal devra sacrifier, avec l'aide de ses camarades d'âge, de nombreux poulets et cinq porcs aux ukin balingabu (traitant les affaires de meurtre), kareñaku (initiation masculine) et karaayaku. Chacun s'attend à l'annonce d'un décès humain dans les proches environs.

Des rituels mis en œuvre dans les affaires de doubles, le kepuleen (cf. note 6) est le plus central. Reprenons l'exemple de cet homme atteint de maux de gorge et que l'on a dirigé vers l'autel des doubles crocodile.

La scène se déroule sous la véranda de la maison, devant l'autel en pisé adossé au mur. Le chef de famille, détenteur du utilaw, prend la noix de bukunumabu que le malade a apportée et une louche en fruit de rônier qu'il remplit de l'eau du canari familial. Il récite tout d'abord la liste des noms de ces prédécesseurs qui, dans le ank, ont entretenu l'autel, puis il appelle :

" Ioniey, toi qui prend à la gorge,

voici notre frère,

il dit que tu l'as pris,

toi qui vis dans l'eau, voici l'eau. "

Il prend la noix, en croque et recrache de petits morceaux sur l'autel. Il donne le reste au malade pour qu'il la croque aussi, la mâche bien et se frotte le corps avec.

N. Diatta (ouvrage à paraître) décrit un autre rituel pratiqué par les Joola Juwaat (région de Diembering), lequel consiste à " laver un malade chez les doubles ". Lorsqu'une personne tombe évanouie, le spécialiste, l'homme qui voit les doubles, vient dans le plus grand secret au moment où la marée est haute. Il verse quelques gouttes d'eau sur le malade, puis 
se retire. Il revient à la marée suivante pour renouveler le même geste, afin de libérer le double de la mangrove où il était prisonnier et le rendre à son milieu maritime.

En dehors du kepuleen, un rituel pratiqué aux mêmes fins que le kajupen ajamaat ou esulelu existerait chez les Felup : il s'agirait toujours de restaurer le principe vital, appelé ici yalorey, dans le corps de la victime, mais en utilisant un arbre au lieu d'un animal domestique. Mais sur ce rituel, très rarement évoqué, l'enquête reste toute entière à mener. Hormis ces deux rituels, les relations entretenues avec les doubles ne semblent guère faire appel à des procédés qui recourraient à des instrumentations intermédiaires, objets, gestes ou paroles. Pour une large part, qu'il s'agisse du rêve envoyé par tel ou tel bákin, ou de la "clairvoyance ", les formes de mise en relation doubles-humains semblent relever d'un mode plutôt médiumnique qu'instrumental.

\section{Le lien double-bumain}

J. D. Sapir rapportait que les Kujamaat affirment qu'un homme et son double communiquent entre eux, chacun ayant pleinement connaissance de ce que fait l'autre. Chez les Felup, comme nous l'avons vu, il n'est pas donné à tout le monde de connaitre même son double principal. A plus forte raison, les actions de tel ou tel double, les dangers qui le guettent ou les lieux où il réside. Seuls de rares " clairvoyants ", dont cette femme du village de Niaban dont l'histoire est racontée ci-dessus, " voient " ou " sentent " leur double " travailler ". Mais " voir " est une chose, intervenir en est une autre.

Sur ce lien ewuum-humain, les informations restent toutefois contradictoires : la conclusion du récit du cultivateur excédé par l'hippopotame laissait entrevoir que le double de ce dernier, ravageur de rizières, n'était pas innocent. Il est mort, dit-on, de ne pas s'être soumis à un jugement public. Malgré l'affirmation générale d'une précession des sizenum sur leurs homologues humains, certains cas mettent en scène des doubles manipulés par leur alter humain. Tel ce vieux que les fermmes, lorsqu'elles partaient à une pêche collective, se devaient d'avertir afin qu'il écarte de leurs nasses les poissons doubles d'habitants du village. Elles partirent un jour, de très bon matin, sans rien dire. Mais il les a entendues et leur a 
crié : " ah !, vous allez au marigot ? Vous me trouverez là-bas ! ". Au moment où elles s'apprêtaient à rentrer dans l'eau, un serpent est sorti des herbes et a mordu l'une d'elles.

Ceux qui ont le pouvoir d'intervenir dans le monde des doubles peuvent ainsi venir au secours d'une personne (sous son apparence humaine ou animale) partie en brousse ou au marigot. Un jeune homme du village de Esana raconte un tel épisode :

* L'autre jour j'étais allé pêcher en pirogue avec un ami. A un moment, une femelle hippopotame avec son petit nous a barré la route. Alors mon ami a commencé à l'insulter de tous les noms. J'ai essayé de le faire taire. L'hippopotame nous a repoussés dans les palétuviers et nous a coincés là toute la nuit. Ensuite elle nous a libérés. A notre retour au village, il y a une femme qui m'a dit : " ton ami a de la chance que tu aies été avec lui, sinon je vous faisais chavirer et je vous aurais noyés!"

Il est quelques détenteurs d'utilaw fort réputés. L'un d'eux explique son travail de la façon suivante :

"Dans ma famille, il y en a d'autres qui voient les doubles. Mais c'est comme à l'école, il y en a un qui est meilleur. Comme j'arrive à séparer des gens (i.e. à arracher des doubles à ceux qui les ont retenus) j'ai plus de forces. Même si les autres vont chercher le double d'une personne qu'on a pris, ils viennent s'adresser à moi : on a cherché le double de tel, le voilà. Et c'est moi qui le remet dans notre concession. Quand je sépare quelqu'un, la nuit, je vois bien. Et quand je me réveille, je dis à ma femme ou à ma fille : 'j'ai séparé tel ou tel, de toutes façons, il viendra'. Ce n'est pas moi qui lui dis de venir ; je l'attend. Il viendra et me dira : 'voilà, je suis allé sacrifier à tel endroit, on m'a dit de venir ici pour que tu me fasses le kepuleen' ".

L'action d'un humain sur un double est fréquemment, si ce n'est pas toujours, médiatisée par l'intervention d'un troisième composant de la personne : yaarori, terme que les Joola francophones traduisent par " silhouette ". Selon l'eschatologie felup, lorsqu'une personne meurt, ses parents défunts viennent " tirer " de son corps cette " silhouette ". Pour donner une approximation de ce qu'est yaarori, les vieux prennent souvent l'image d'un couteau qu'on dégaine : à la mort, yaarori sort du corps comme le couteau sort de sa gaine. Tandis que le corps reste sur l'estrade funéraire avant de " s'écrouler ", yaarori, que les parents défunts 


\section{Odile Journet}

ont mise debout, devient " apur ñotaw (sortir/marcher). C'est elle que l'on peut voir sur le chemin qui sort du village tandis que se déroulent les danses funéraires. Le défunt part avec les yaarori du riz, du bétail, des pagnes, des perles qu'il possédait. Le monde des morts est l'exacte reduplication de celui des vivants, ukin et siwuum compris qui y vivent en tant que " silhouettes".

En général, yaarori est le principe impliqué dans les affaires de sorcellerie : le sorcier (asayo) ou, plutôt, le yaarori du sorcier, emprunte l'apparence d'une autre personne ou d'un animal pour s'emparer du yaarori de sa victime qu'il transforme en animal à des fins de dévoration. Mais yaarori intervient aussi dans les affaires de doubles.

Chaque ewuum a un yaarori, chaque humain en possède de quatre à six dont tous, sauf un, sont des simulacres.

Bien qu'aucun informateur ne m'ait livré de théorie définitive et univoque à ce sujet, yaarori semble intervenir de deux manières : il se " pose " sur le double avec lequel ego a un rapport privilégié ; ou bien, s'il s'agit d'un sorcier, son yaarori, sous une apparence quelconque, vient appeler quelqu'un, le désignant ainsi au yaarori d'un double animal qui l'emportera quelques heures ou quelques jours plus tard, dans les profondeurs du marigot.

Que ce soit par le biais du rêve, de yaarori, de la clairvoyance ou encore de certains ukin, les relations doubles à doubles laissent toujours place, a priori ou a posteriori, à une intervention de leurs homologues humains. Ainsi ce vieux, qui a perdu un œil, explique : " Est-ce que je suis né borgne ? Non, c'est le double de mon oncle paternel qui avait attaqué le mien dans le marigot. Pour sauver mon deuxième œil, ma mère a demandé à l'oncle de faire le kepuleen. "

\section{Le monde de bèkènabu}

Dans les exemples ajaamat et esulelu, nous avons vu que les relations humains/doubles, double/double, etc, s'exerçaient par le biais d'une communauté de résidence, les doubles habitant dans l'enclos ou dans la brousse proche de la maison. Dans l'exemple felup, la partition humains/doubles se développe en une partition générale de l'espace : esukey (village) / bèkènabu (monde des doubles). 


\section{Un monde diffracté}

Les doubles ne résident pas dans l'espace social des humains, mais c'est cet espace lui-même qui a son envers ou son double dans le monde des siwuum.

Bèkènabu est toujours situé à une certaine distance du village ou des rizières, en forêt ou dans les profondeurs du marigot ou de la mer. Deux espaces qui, en général, ne s’interpénètrent pas. De même que les doubles de forêt ne se laissent guère chasser, ceux de l'eau ne sont pêchés qu'exceptionnellement : il faut pour cela qu'ils aient quitté les bas-fonds et leurs semblables, ce qui est rare sauf, dit-on, lorsque leur alter humain est complètement ivre. Mais si les doubles felup ne s'aventurent guère dans le village humain, à la différence des sauriens et serpents kujaamat et esulelu, les humains, eux, ont affaire à la brousse, qu'ils la fréquentent pour pêcher, chasser, ou rejoindre des rizières éloignées. Lieu des rencontres sidérantes avec un génie ou un bákin, lieu d'éventuels guetapens, lieu d'errance des revenants, la brousse (la forêt, le marigot) est toujours inquiétante même pour ceux qui en sont les plus familiers. C'est là qu'interviennent, pour protéger les villageois ou pour s'en défendre, les siwuum.

Dans ce monde parallèle qu'est bèkènabu, la relation humain/animal, prolongement de l'opposition générale village/brousse, prend la forme d'une inversion symétrique :

* Tu vois par exemple un crocodile qui n'est pas un ewuum : il entrấnera sa victime dans les palétuviers où il la mangera. Mais un crocodile ewuum l'emmènera dans les profondeurs. Là-bas, c'est comme sur la terre, ils vivent en famille. Ils sont là comme nous qui sommes en train de bavarder. S'ils t'emmènent, les uns voudront t'épargner, mais les autres refuseront, car maintenant tu les auras découverts. Alors ils t'égorgent et te dépècent comme un animal. "

Dans bèkènabu, les siwuum vivent regroupés en maisons, par espèces, à l'instar de leurs homologues humains. Ou, plus exactement, l'espace social felup est démultiplié en autant de "maisons" que les membres de chaque ank peuvent avoir de doubles. Ces " maisons" sont loin de correspondre à la réalité sociale de la division d'un quartier ou d'un village en siank. Et ce, pour deux raisons que nous avons évoquées et qui se conjuguent : le ank, unité de co-résidence, ne rassemble pas les membres d'un même lignage ; les cohabitants humains d'un ank n'ont 
pas un même patrimoine de doubles. Lorsqu'un homme se marie, on dit que ses doubles vont chercher ceux de sa femme (tout au moins ceux qu'elle possède en commun avec lui) pour les ramener dans leurs maisons communes. Mais cette opération peut être mise à mal par d'autres doubles qui auront rapté et retenu prisonnier tel ou tel, ou encore l'auront caché en un lieu inaccessible. S'ensuivent alors des désordres dans la destinée de son homologue humain (stérilité, troubles du comportement). Cette supposition est fréquente lorsqu'il s'agit d'un remariage après divorce, les doubles de la famille du premier mari retenant, cachant ceux de la femme.

Ainsi les relations sociales entre humains sont-elles sous la dépendance non pas de l'organisation d'un univers symétrique ou parallèle, mais de l'agencement - plus ou moins heureux - de leurs multiples appartenances dans un monde où elles sont révélées, disséquées et recombinées. Bèkènabu est le lieu où pourraient se lire, de manière topographique, les éléments idéaux d'une harmonie individuelle et collective jamais atteinte.

Réflexions provisoires sur ce système

Que la personne soit constituée de multiples composants n'a certes rien d'original au regard des sociétés africaines. Ce "feuilletage " (F. Héritier, 1983 : 65) emprunte chez les Felup la figure suivante : ajamaataw, la personne, est constituée d'un corps (enil), d'une conscience (bubinum), d'un capital de force (sembe), d'une " silhouette" (yaarori), accompagnée de ses trois à cinq simulacres, et de quatre à six doubles animaux (siwurm).

Qu'un enfant puisse avoir plus d'affinités avec d'autres parents que ceux de son patrilignage est un fait légitimé par le système des siwuum: " il y a des enfants qui se détachent de leurs parents, qui ne restent pas dans leur ankaw. Ils ont un double autre part et ils vont là-bas. Il faut les laisser faire. "Ce fait psychologique peut être rapproché des théories de la conception : un enfant qui naît réincarne le yaarori d'un défunt qui, au 
Exemple felup (schéma simplifié)

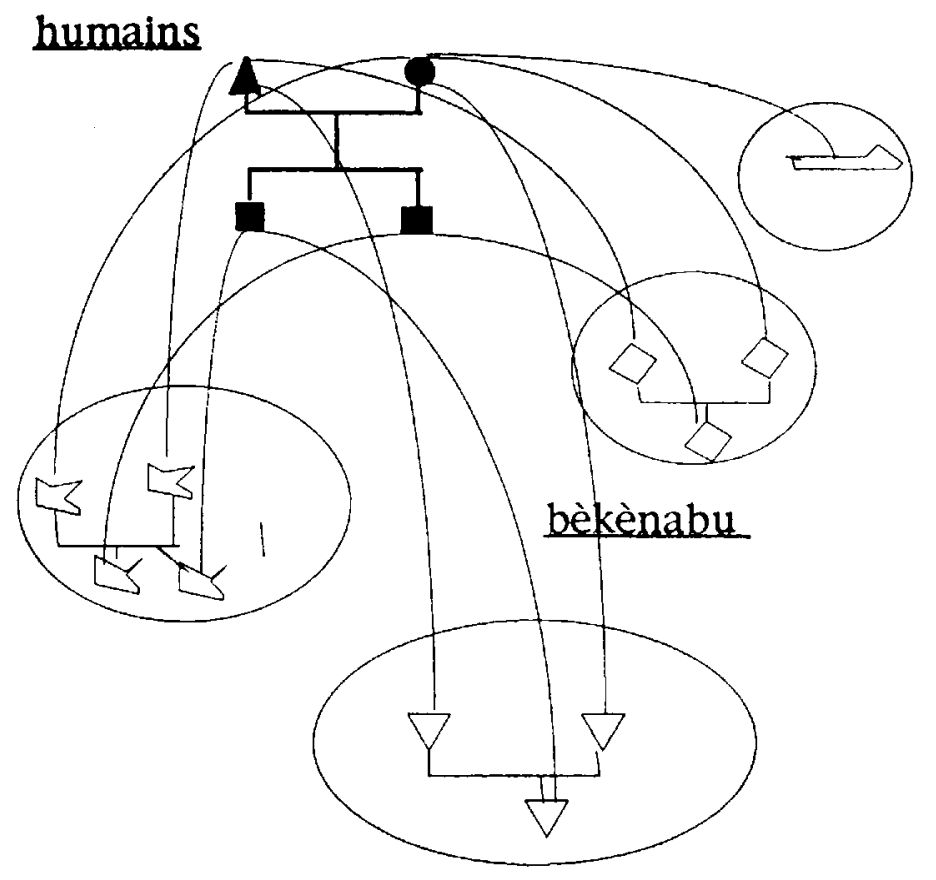


terme de multiples épisodes, revient sous la forme de l'awna - * âme " de l'enfant à nâtre. L'enfant peut être porteur du yaarori de n'importe quel ancêtre du côté paternel ou maternel. Mais il peut aussi venir d'autres lignages, en suivant par exemple la voie de tel ou tel bákin auquel il est resté attaché. Lorsque yaarori revient, on dit qu'il est accompagné par un bákin villageois auquel il était, dans sa vie antérieure, particulièrement lié ("c'est tel bákin qui m'a fait renaître " disent souvent les vieux). Une fois raccompagné dans le quartier ou le sous-quartier où est implanté le bákin en question, c'est le utilaw de telle ou telle maison qui servira en quelque sorte de point d'attraction : yaarori, devenu awua, attiré par ce utilaw, ira se fixer dans le ventre d'une épouse du lignage. Le système des siwuum, tout en recomposant des chaînes de filiation, propose un modèle original d'individualisation : un enfant peut avoir pour double principal ce qui n'était, pour ses géniteurs, qu'un double secondaire hérité, par des voies diverses, de parents éloignés. L'opposition entre catégories sociales ou segments de filiation est ici intégrée au sein même de la personne. Il y en a en felup un mot pour dire "celui qui est écarté entre plusieurs parents" (jitangol).

Mais l'exemple felup complique le schéma des deux premiers cas (Kujaamat et Esulelu) en intégrant au moi des appartenances plus éloignées, mais surtout méconnues a priori. Aucune règle systématique ne préside à la constitution de ce stock de doubles qui composent un individu. Au contraire, toutes les histoires de doubles mettent en scène une quête aléatoire, quête de doubles cachés par mauvaise intention, ou errants à la lisière de bèkènabu. Identifier un double inconnu ou égaré, source d'afflictions diverses, et le retrouver, est une préoccupation courante. D'une union stérile, on commencera par imputer la cause à une dispersion des doubles des conjoints. Lorsque les femmes spécialistes du rituel kañaalen, destiné à préserver leur descendance, se réunissent, elles sacrifient et "envoient " leurs doubles respectifs, chaque espèce de son côté, à la recherche du (des) double(s) de la femme qui perd ses enfants. Plus exactement, elles vont sacrifier à différents ukin capables de leur montrer le chemin par la voie du rêve :

"Ici quand une femme se confie à vous, c'est parce que quelqu'un l'a prise et l'a emmenée quelque part (i.e. "a pris son double"). Ici le 
plus souvent, on l'emmène dans la mer et on a beau chercher, on n'arrive pas à la trouver ".

* L'autre fois, je vous ai dit ce que j'avais vu en rêve. Tout Esana s'était réuni. On l'avait trouvée dans le puits et on l'avait tirée du puits pour la faire sortir. Je vous avais dit : " les enfants, il faut crier ! ”. Vous aviez détaché vos pagnes et dansé, et vous aviez dit : " oui, nous sommes soulagées. Naylo nous a indiqué où aller, dans les kutilako (pl. de utilaw)"

Perdu dans ce jeu complexe d'images en miroir, on peut enfin se demander ce que le système des doubles nous dit des représentations joola de la division du monde social. L'espace social felup, dont nous n'avons parlé jusqu'alors que sous l'aspect de la division entre unités de résidence, est un espace avant tout marqué dans l'histoire locale par une série de déchirements et de morcellements. Ces déchirements, dont le souvenir hante les villageois, sont en grande partie liés aux guerres intervillageoises et à leur mode de conclusion : les villages vaincus étaient rasés, leurs habitants survivants et leurs autels lignagers capturés et répartis entre les siank du village vainqueur. Les grands ukin qu'on ne pouvait déplacer, on en confiait l'entretien et la responsabilité à des prêtres du camp vainqueur. Les enfants des vaincus, ainsi " adoptés ", étaient coupés de la connaissance de leurs ascendances (et donc de la connaissance de leurs droits sur les terres que les vainqueurs s'étaient appropriées). D'autres occasions de dispersion peuvent être évoquées : la pratique courante du " déguerpissement thérapeutique " (lorsque l'on souffre, soi, son conjoint ou ses enfants, d'une maladie chronique, on déménage dans un autre quartier, voire un autre village) ; la mort d'un chef de famille avec des enfants jeunes (ils sont partagés et dispersés) ; et enfin la guerre de libération nationale qui laisse de profondes divisions entre ceux qui se sont battus du côté des Portugais et ceux qui ont combattu avec le PAIGC (Parti Africain de l'Indépendance de la Guinée et des Iles du Cap Vert). Le thème de la dispersion est un thème récurrent en particulier dans tous les chants de funérailles ou de lutte.

"Récupérer " le double caché, kidnappé ou errant, faire en sorte qu'il soit pris en charge au bon endroit de bèkènabu, autant d'opérations qui visent en quelque sorte à recomposer, dans l'espace des doubles, un 
espace social ou plus exactement des chaînes de filiation qui, du côté des humains, ont été brisées.

Appliquée aux fait felup, la réflexion de Lévi-Strauss - "Le totémisme se ramène à une façon particulière de formuler un problème général : faire en sorte que l'opposition au lieu d'être un obstacle à l'intégration, serve plutôt à la produire " (1991 : 132) - pourrait conduire à développer, dans la même ligne ou à son encontre, quelques hypothèses interprétatives.

L'intégration, entendue au sens sociologique, se réalise ici par la volonté permanente, sans cesse réaffirmée, de l'unité villageoise, mais aussi des unités de résidence, quartiers, sous-quartiers et siank. A Esana, il est interdit de dire publiquement : " je viens de tel village " ou : * toi, tu viens de... " sous peine d'être obligé de sacrifier au bákin de l'initiation masculine. Chacune de ces unités locales - ankaw, ebandey (réunion de deux à quatre siank), sous-quartier, quartier, village - est dotée d'un nom, de moult surnoms, chacune a ses propres chants, chacune est liée aux autres par des relations d'alliance politique et d'entraide, chacune intervient en tant que telle dans les rituels collectifs qu'il s'agisse des luttes, des funérailles, de l'initiation, des mariages, des chasses collectives, etc. Par les danses, les déambulations, les cortèges, l'ordonnancement des rencontres entre délégations, etc., ces rituels ont en commun d'organiser une mise en scène aussi spectaculaire que minutieuse des cadres d'appartenance locale quelle que soit leur composition interne. D'autres procédures concourent à un quadrillage de l'espace essentiellement fondé sur ces unités, en particulier les modes de transmission d'un certain nombre d'ukin qui, à la mort de leur détenteur, doivent « tourner " selon un mouvement immuable entre les différents sous-quartiers du village. De manière générale, les liens du sol semblent l'emporter sur les groupes de filiation. Filiation qui peut elle-même être subvertie par les liens de corésidence : lorsqu'un lignage s'éteint, les "adoptés" du ankaw en reprennent les rizières, le nom et les autels lignagers. Face à ce puissant mécanisme d'intégration locale, le système des siwuum permet de recomposer, à travers les segmentations et les dispersions, des appartenances lignagères souvent occultées par les avatars de l'histoire locale. Mais il 
réintroduit toutes les oppositions séculaires que le système villageois s'efforce, avec tant d'opiniâtreté, de gommer.

De manière générale, le système des siwuum met fortement l'accent sur les problèmes de résidence et plus particulièrement la variante felup avec l'idée de bèkènabu. Remettre chacun et les composantes de chacun à leur juste place est un travail sans fin. Si tant est que l'« idiome siwuum " puisse jouer le rôle d'opérateur totémique, ce n'est pas tant en ce qu'il permet d'avoir recours aux divisions des espèces animales, mais à celles de l'espace bèkènabu, c'est-à-dire à des divisions spatiales et plus précisément, des divisions résidentielles qui proposent un modèle idéal de congruence entre parenté, affinités, et cohabitation. La littérature ethnologique a peut-être accordé moins d'importance à la question de la résidence qu'à celles de la filiation, de l'alliance ou des systèmes d'appellation. L'exemple joola invite à reconsidérer cette dimension. Les liens de co-résidence sont toujours pensés comme fortement problématiques comme en témoignent par exemple les attaques en sorcellerie entre agnats ou entre belles-sœurs vivant dans un même ankaw, ou encore les va-et-vient entre deux siank que l'on peut observer dans une lignée de deux ou trois générations.

Le modèle felup du double animal semble ainsi se situer à l'interférence de trois logiques : celle de la différenciation des lignages par les autels de doubles, celle du cycle vital, celle, enfin, des appartenances sociales et résidentielles qui sont ici marquées du sceau de l'aléatoire et d'une perpétuelle insatisfaction.

Odile Journet Université de Lyon II 
Références bibliographiques

Diatta, $\mathrm{N}$.

"Les Joola : proverbes et expressions " (dicton 3-3), ouvrage à paraitre aux éditions Karthala.

Dupire, $\mathrm{M}$.

1991 "Totems sereer et contrôle rituel de l'environnement ", L'Homme, avril-juin, 118, XXXI, 2, 37-66.

Héritier, $\mathrm{F}$.

1983 "L'identité samo ", in C. Lévi-Strauss, L'identité, Paris, PUF. Lévi-Strauss

1991 Le totémisme aujourd'hui, PUF, (lère éd. 1962)

Sapir, E.

1977 "Fecal animals : an example of complementary totemism ", Man, (N.S.) $12,1-21$

Smith, P.

1973 "Principes de la personne et catégories sociales", in La notion de personne en Afrique Noire, Paris, éd. CNRS 